OPEN ACCESS

Edited by:

Xin-Wu Cui,

Huazhong University of Science and

Technology, China

Reviewed by:

Sikandar Shaikh,

Shadan Hospital and Institute of

Medical Sciences, India

Li Ping Sun,

Tongji University, China

*Correspondence:

Fang Nie

ery_nief@/zu.edu.cn

Specialty section:

This article was submitted to

Cancer Imaging and Image-directed Interventions,

a section of the journal

Frontiers in Oncology

Received: 31 January 2021 Accepted: 26 April 2021

Published: 26 May 2021

Citation:

Wang Y, Dong T, Nie F, Wang G, LiU T and Niu Q (2021) Contrast-Enhanced Ultrasound in the Differential Diagnosis and Risk Stratification of ACR TI-RADS

Category 4 and 5 Thyroid Nodules With Non-Hypovascular.

Front. Oncol. 11:662273. doi: 10.3389/fonc.2021.662273

\section{Contrast-Enhanced Ultrasound in the Differential Diagnosis and Risk Stratification of ACR TI-RADS Category 4 and 5 Thyroid Nodules With Non-Hypovascular}

\author{
Yanfang Wang ${ }^{1}$, Tiantian Dong ${ }^{1}$, Fang Nie ${ }^{1 *}$, Guojuan Wang ${ }^{1}$, Ting Liu ${ }^{1}$ and Qian $\mathrm{Niu}^{2}$ \\ ${ }^{1}$ Medical Center of Ultrasound, Lanzhou University Second Hospital, Lanzhou, China, ${ }^{2}$ Department of Pathology, Lanzhou \\ University Second Hospital, Lanzhou, China
}

Objective: This study aims to investigate the value of contrast-enhanced ultrasound (CEUS) in the differential diagnosis and risk stratification of ACR TI-RADS category 4 and 5 thyroid nodules with non-hypovascular.

Methods: From January 2016 to December 2019 in our hospital, 217 ACR TI-RADS category 4 and 5 nodules with non-hypovascular in 210 consecutive patients were included for a derivation cohort. With surgery and/or fine-needle aspiration (FNA) as a reference, conventional ultrasound (US) features and CEUS features were analyzed. Multivariate logistic regression analysis was used to screen the independent risk factors and establish a risk predictive model. Between January 2020 and March 2021, a second cohort of 100 consecutive patients with 101 nodules were included for an external validation cohort. The model was converted into a simplified risk score and was validated in the validation cohort. The area under the receiver operating characteristic curves (AUC) were used to assess the models' diagnostic performance.

Results: Micro-calcification, irregular margin, earlier wash-out, centripetal enhancement, and absence of ring enhancement were independent risk factors and strongly discriminated malignancy in the derivation cohort (AUC $=0.921,95 \% \mathrm{Cl} 0.876-0.953$ ) and the validation cohort $(0.900,0.824-0.951)$. There was no significant difference $(P=0.3282)$ between the conventional US and CEUS in differentiating malignant non-hypovascular thyroid nodules, but a combination of them (the predictive model) had better performance than the single method (all $P<0.05$ ), with a sensitivity of $87.0 \%$, specificity of $86.2 \%$, and accuracy of $86.6 \%$ in the derivation cohort. The risk score based on the independent risk factors divided nonhypovascular thyroid nodules into low-suspicious (0-3 points; malignancy risk <50\%) and high-suspicious (4-7 points; malignancy risk $\geq 50 \%$ ), the latter with nodule $\geq 10 \mathrm{~mm}$ was recommended for FNA. The risk score showed a good ability of risk stratification in the validation cohort. Comparing ACR TI-RADS in screening suitable non-hypovascular nodules for FNA, the risk score could avoid $30.8 \%$ benign nodules for FNA.

Conclusions: CEUS is helpful in combination with conventional US in differentiating ACR TI-RADS category 4 and 5 nodules with non-hypovascular. The risk score in this study has 
the potential to improve the diagnosis and risk stratification of non-hypovascular thyroid nodules.

Keywords: thyroid nodules, thyroid imaging reporting and data system, ultrasonography, contrast media, fine-needle aspiration, risk stratification

\section{INTRODUCTION}

The widespread use of thyroid ultrasound (US) has increased the number of asymptomatic thyroid nodules that can be detected $(1,2)$. However, only $5 \%$ to $15 \%$ of thyroid nodules are malignant $(3,4)$. The main challenge in the evaluation of thyroid nodules is to identify those malignant nodules from benign ones. Though the conventional US is considered the preferred imaging method and valuable for the differential diagnosis of thyroid nodules, overlaps can occur between benign and malignant thyroid nodules, thus reducing the diagnostic accuracy of thyroid nodules (5). Fineneedle aspiration (FNA) is an accurate and effective method in the qualitative diagnosis of thyroid nodules preoperatively. However, at least half of all biopsied nodules are benign nodules (6), and up to $30 \%$ with indeterminate cytology results $(7,8)$. Therefore, overdiagnosis and overtreatment of benign thyroid nodules may have occurred. Thus, new techniques improving the diagnostic accuracy of thyroid nodules while reducing the number of unnecessary FNA are required.

Since the European Federation of Societies for Ultrasound in Medicine and Biology (EFSUMB) released the Guidelines and recommendations for the hepatic use of contrast-enhanced ultrasound (CEUS), CEUS has been used for non-hepatic applications because of its advantages in visualizing the microcirculation and the dynamic enhancing process of tumors $(5,9,10)$. Previous studies have shown the good performance of CEUS in differentiating benign and malignant thyroid nodules and screening appropriate nodules for FNA, as an effective supplement technique of conventional US (11-13). And the diagnostic accuracy of thyroid nodules can be increased by combining conventional US and CEUS $(14,15)$.

Hypo-enhancement on CEUS is considered the most precise predictor of malignancy for thyroid nodules with high sensitivity, specificity, and accuracy of $82 \%, 85 \%$, and $84 \%$, respectively (5). While iso-/hyper-enhancement on CEUS can suggest benignity $(16,17)$. However, in our clinical practice, one-third of nodules with American College of Radiology's Thyroid imaging reporting and data system (ACR TI-RADS) (18) category 4 and 5 on conventional US appear iso-/hyper-enhancement on CEUS (we defined as "non-hypovascular thyroid nodules") that turn out to be malignant. Thus, the differential diagnosis and risk estimation for non-hypovascular thyroid nodules are challenging.

To our knowledge, prior studies paid little attention to the differential diagnosis and risk estimation of non-hypovascular thyroid nodules. In this study, based on preoperative conventional US features and CEUS features, we attempt to establish a predictive model and develop a risk score to differential diagnose and stratify non-hypovascular thyroid nodules, hoping to provide some useful information for clinical decision-making of non-hypovascular thyroid nodules.

\section{MATERIALS AND METHODS}

\section{Patients}

This retrospective study was conducted under the Declaration of Helsinki. Ethical approval was obtained from the Ethics Committee of Lanzhou University Second Hospital. All patients signed informed consent before CEUS examination. In clinical daily work, we usually suggest nodules with indeterminate or suspicious diagnosis after conventional US as target nodules for CEUS. Consecutive patients who presented to our hospital for thyroid nodules and received both conventional US and CEUS were included in this study. The inclusion criteria were as follows: (a) ACR TI-RADS category 4 and 5 nodules with greater than or equal to $10 \mathrm{~mm}$ in the largest diameter; (b) nodules with complete conventional US and CEUS imaging materials; (c) nodules with isoenhancement or hyper-enhancement on CEUS; (d) confirmed by surgery or FNA. The exclusion criteria were as follows: (a) large nodules without perinodular normal parenchyma as a reference; (b) nodules with Bethesda category II not confirmed by repeated FNA or found a change in size and ACR TI-RADS category during the at least 1 year's follow-up. For the derivation cohort, 210 consecutive patients with 217 nodules were included between January 2016 to December 2019, a second cohort of consecutive 100 patients with 101 nodules between January 2020 to March 2021 were included for the external validation. A total of 310 patients $(90$ males and 220 females, mean age, $48.4 \pm 12.4$ years; range, 18 - 76 years) were included in this study. The diameter of 318 nodules was $17.5 \pm 7.3 \mathrm{~mm}$ (size range $10.0-40.0 \mathrm{~mm}$ ).

\section{Conventional Ultrasound}

Two radiologists with more than 5 years' experience in conventional US and at least 3 years' experience in CEUS performed the ultrasound examinations. They received standardized training and had experience of 50 thyroid nodules before collecting materials in this study. Conventional US images of thyroid nodules were obtained using an iU22 scanner (Philips Medical Systems, Bothell, USA) equipped with a 5- to $12-\mathrm{MHz}$ linear probe and the ACUSON Sequoia scanner (Siemens Medical Solutions, Michigan, USA) with a 4- to $10-\mathrm{MHz}$ linear probe. The ACUSON Sequoia scanner was not used in the derivation cohort. The patient was placed on a bed in the supine position and the neck region was fully exposed. Conventional US static images of thyroid nodules were acquired by carefully scanning the thyroid and adjacent tissues both transversely and longitudinally. The images were stored on the instrument's internal hard drive for further analysis.

On the conventional US, the thyroid nodules were evaluated according to the following features: size (maximum diameter), solid or almost completely solid composition (no or yes); echogenicity (hyperechoic/isoechoic or hypoechoic/very hypoechoic); shape (wider-than-tall or taller-than-wide); margin (regular-smooth/ill- 
defined or irregular-lobulated/irregular/extrathyroidal extension); punctate echogenic foci/micro-calcification (absence or presence); and vascularity (19) (type 1, no vascularity-defined as no power Doppler flow in the periphery or within the nodule; type 2, peripheral vascularity-defined as power Doppler flow only in the periphery of the nodule; and type 3, intranodular vascularitydefined as power Doppler flow within the nodule regardless of power Doppler flow in the periphery of the nodule).

\section{Contrast-Enhanced Ultrasound}

Contrast-enhanced ultrasound (CEUS) examination was conducted using the same instrument as the conventional US with a 3- to $9-\mathrm{MHz}$ linear probe of $\mathrm{iU} 22$ or a 4 - to $10-\mathrm{MHz}$ linear probe of Sequoia and the same radiologists. CEUS was performed using a low mechanical index $(\mathrm{MI}=0.06)$ to minimize the destruction of microbubbles and the loss of artificial signals. The plane with the maximum nodular size and an appropriate amount of surrounding parenchyma was selected in each nodule for CEUS. Patients were instructed to stop swallowing and to breathe calmly throughout the process. The contrast agent (SonoVue, Bracco, Milan, Italy) was mixed with $5 \mathrm{ml}$ of saline until a homogeneously mixed suspension was obtained. Then, 1.8 to $2.0 \mathrm{ml}$ of the suspension was rapidly pushed into the patient's antecubital vein via a 20/22-gauge probe while their body position remained unchanged. The US machine's timer was activated while the contrast agent was injected. Each contrast imaging acquisition lasted for at least two continuous minutes, and the process was preserved on the instrument's internal hard drive.

On CEUS, the thyroid nodules were evaluated for the following characteristics: homogeneity of enhancement was classified as homogeneous or heterogeneous; enhanced intensity (the perinodular normal thyroid parenchyma as a reference) was classified as iso-enhancement (Figures 1D and 2D), hyper- enhancement (Figures 3D and 4D), hypo-enhancement (excluded from this study), or no-enhancement (excluded from this study); ring enhancement (any regular hyper-enhanced or hypo-enhanced rim in the periphery of a nodule at the peak time) was classified as present (regular and complete) or absent (none, irregular, or incomplete); enhanced border (the boundary between the nodule and the surrounding parenchyma at the peak intensity) were classified as well-defined or ill-defined; centripetal enhancement (the contrast agent enters the nodule from the periphery of the nodule to the center) was classified as yes or no; the relative wash-in time (the time when the contrast agent entered the nodule, compared with the perinodular normal thyroid parenchyma) was classified as earlier, synchronous, or later; and the relative wash-out time (the time when the contrast agent washed out from the nodule, compared with the perinodular normal thyroid parenchyma) was classified as earlier, synchronous, or later.

Conventional US static images and CEUS cine clips were successively reviewed retrospectively by two experienced radiologists (T.T. D. and T. L.) who were blinded to the patients' clinical information and pathology results. The two radiologists analyzed the conventional US and CEUS materials independently first and then reviewed the case with discrepancies, a consensus was reached in those cases after discussion.

\section{Pathological Diagnosis}

In this study, all malignant nodules were confirmed by surgery, benign nodules were confirmed by surgery or FNA, and histological pathology was the final reference if both were performed. A final diagnosis of benignity was made when nodules with Bethesda category II were confirmed by repeated FNA or were found no change in size and ACR TI-RADS category during at least 1 year's follow-up. All of the specimens were categorized by experienced pathologists (Q. N.) who were blinded to the patients' medical history and sonographic findings.
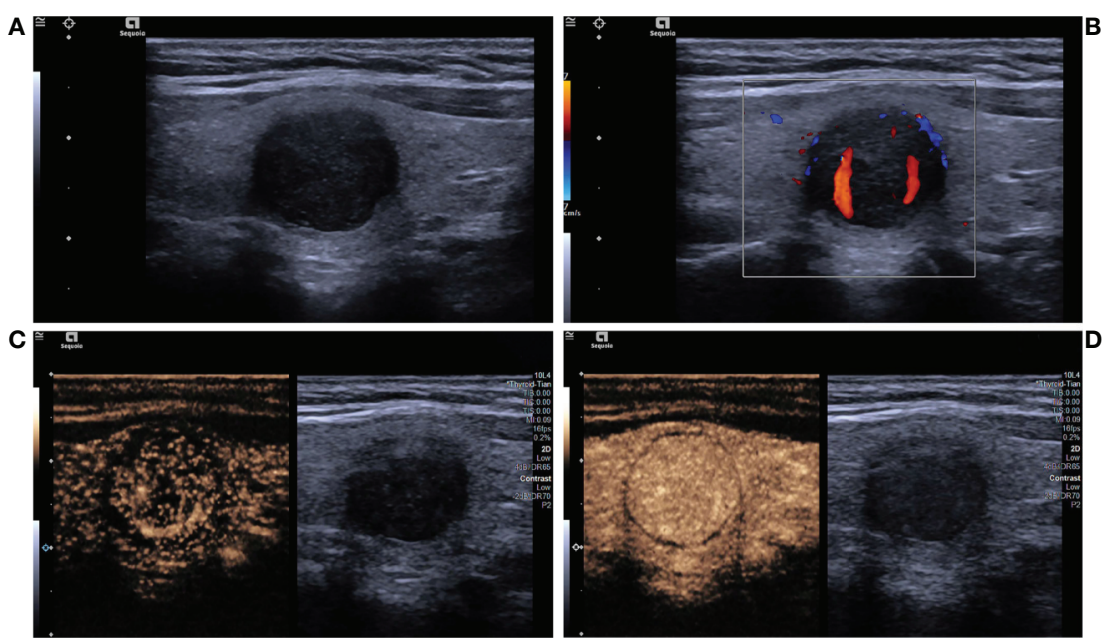

FIGURE 1 | A nodular goiter with adenomatous hyperplasia in a 46-year-old woman. (A) Greyscale ultrasound showed that there was a solid very hypoechoic nodule in the right lobe of the thyroid, with regular margin, wider-than-tall shape, and punctate echogenic foci. The nodule was ACR TI-RADS category 5. (B) Color Doppler showed intranodular and peripheral vascularity. (C) Contrast-enhanced ultrasound revealed diffused enhancement within the nodule at the time of the 11 th second after the injection of contrast agent. (D) Contrast-enhanced ultrasound revealed iso-enhancement at peak (the 16th second after the injection of contrast agent), with regular hypo-enhanced ring. 

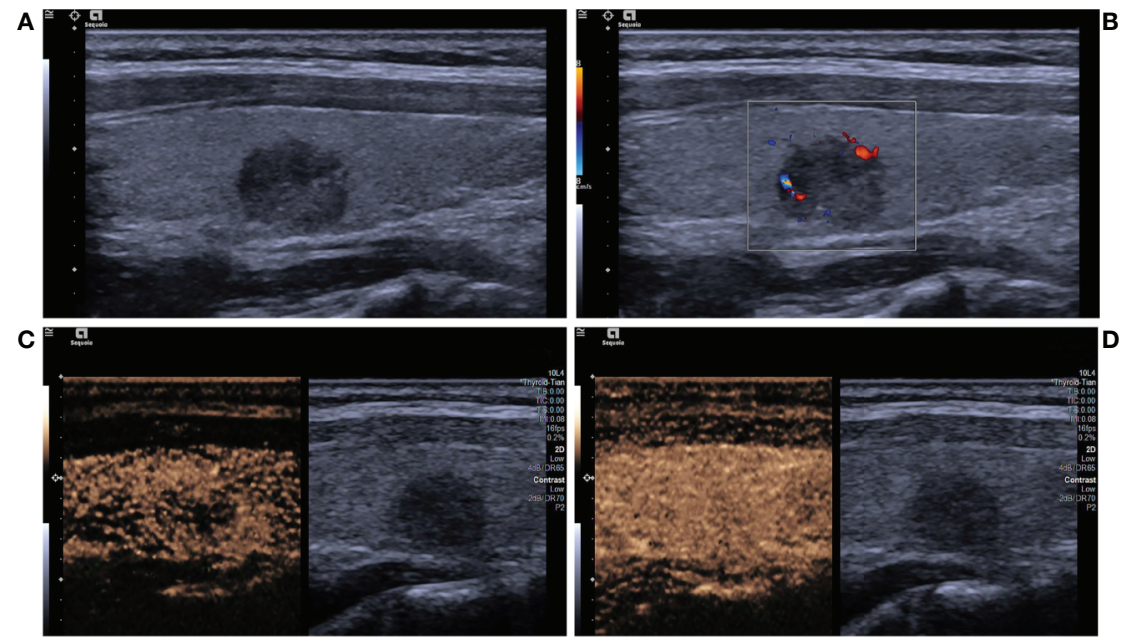

FIGURE 2 | A papillary thyroid carcinoma in a 25-year-old girl. (A) Greyscale ultrasound showed that there was a solid hypoechoic nodule in the right lobe of the thyroid, with irregular margin, wider-than-tall shape, and punctate echogenic foci. The nodule was ACR TI-RADS category 5. (B) Color Doppler showed peripheral vascularity. (C) Contrast-enhanced ultrasound revealed the trend that contrast agent enters the nodule from the periphery to the center (centripetal enhancement) at the time of the 14th second after the injection of contrast agent. (D) Contrast-enhanced ultrasound revealed iso-enhancement at peak (the 22th second after the injection of contrast agent), with ill-defined enhanced border and absence of a regular enhanced ring.
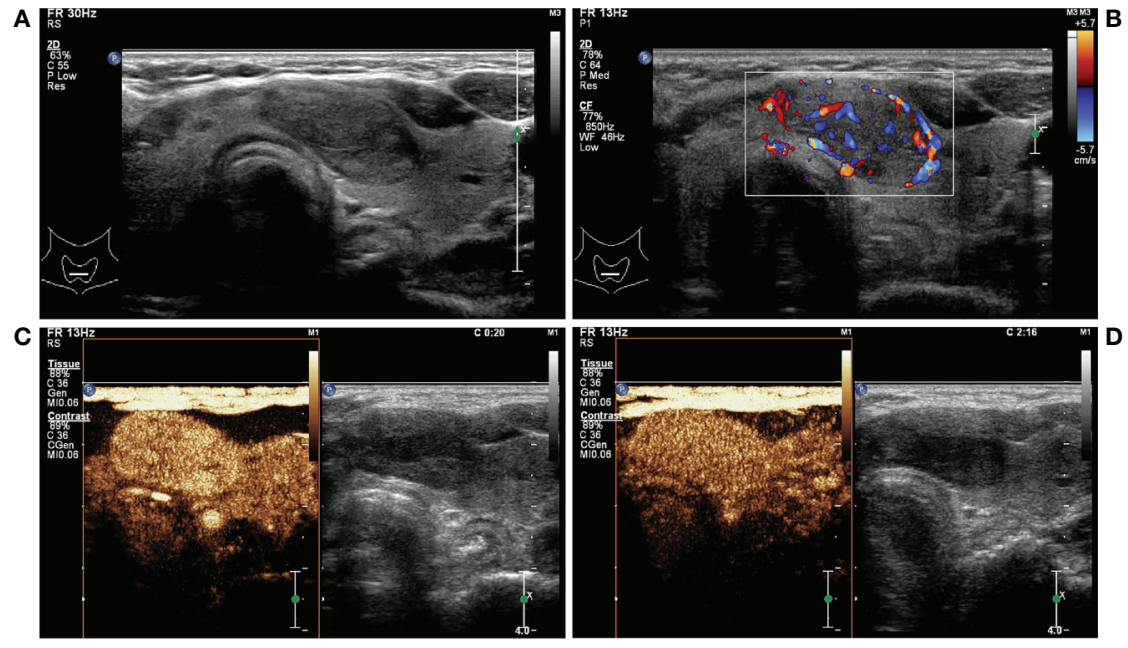

FIGURE 3 | A follicular adenoma in a 63-year-old woman. (A) Greyscale ultrasound showed that there was a solid hypoechoic nodule in the isthmus of the thyroid, with regular margin, wider-than-tall shape, without calcification. The nodule was ACR TI-RADS category 4. (B) Color Doppler showed intranodular and peripheral vascularity. (C) Contrast-enhanced ultrasound revealed hyper-enhancement at peak (the 20th second after the injection of contrast agent), with regular hyperenhanced ring and well-defined enhanced border. (D) Contrast-enhanced ultrasound revealed hyper-enhancement at the 136th second after the injection of contrast agent, which indicates later wash-out than the surrounding thyroid parenchyma.

\section{Model Establishment and Risk Score Construction}

Stepwise logistic regression was used to select variables from 13 ultrasonographic features of non-hypovascular thyroid nodules in the derivation cohort, with pathology as the outcome. The predictive model based on significant variables will model the chance of the malignancy of thyroid nodules, with the formula given by (20-22):

$$
\log \left(\frac{\pi}{1-\pi}\right)=\beta_{0}+\beta_{1} X_{1}+\beta_{2} X_{2}+\ldots \beta_{m} X_{m}
$$

where $\pi$ is the probability of malignancy, $\beta_{0}$ is a constant, and $\beta_{i}$ is the regression coefficient, and the $X$ explains all of the significant variables.

The regression coefficient of each variable in the final model was divided by the absolute value of the smallest regression coefficient and rounded to the nearest integer. Thus, each variable was assigned 

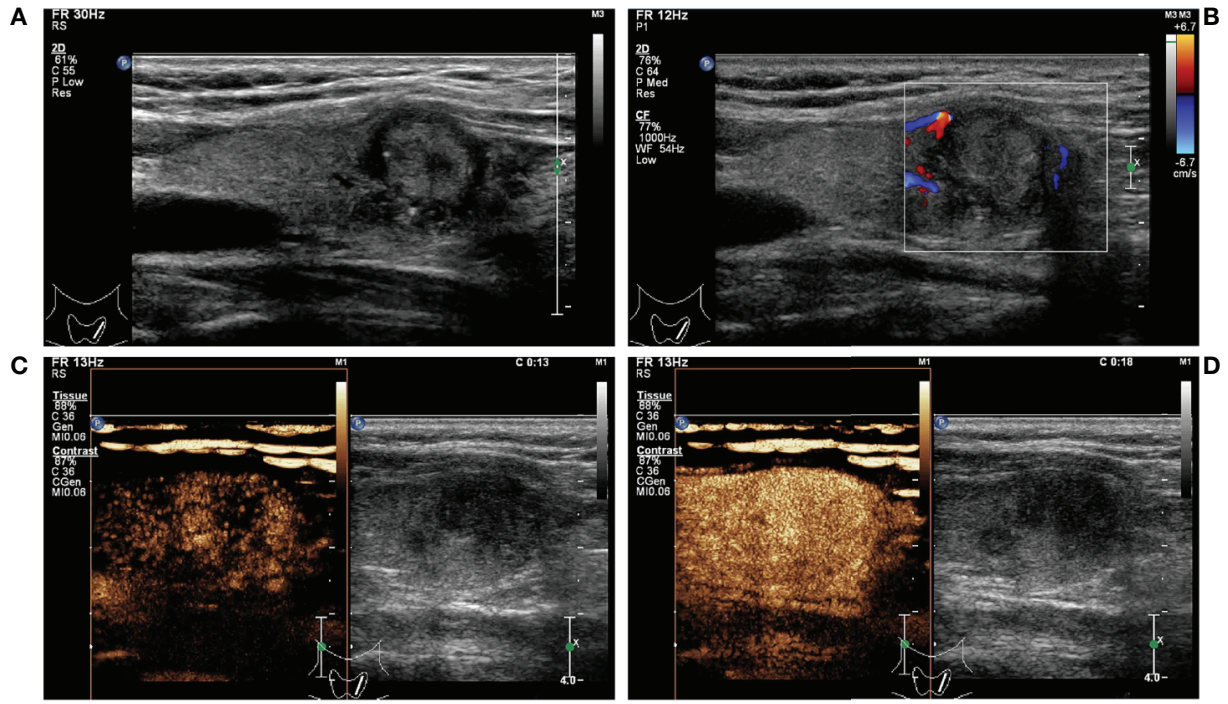

E

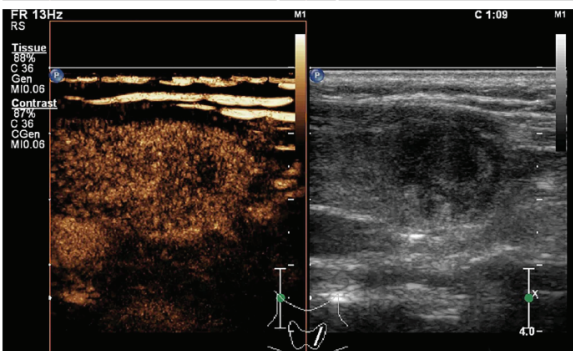

FIGURE 4 | A follicular variant of papillary thyroid carcinomas in a 53-year-old woman. (A) Greyscale ultrasound showed that there was a solid isoechoic nodule in the left lobe of the thyroid, with irregular hypoechoic halo, irregular margin, wider-than-tall shape, and punctate echogenic foci. The nodule was ACR TI-RADS category 5. (B) Color Doppler showed peripheral vascularity. (C) Contrast-enhanced ultrasound revealed the trend that contrast agent enters the nodule from the periphery to the center (centripetal enhancement) at the time of the 13th second after the injection of contrast agent. (D) Contrast-enhanced ultrasound revealed heterogenous hyper-enhancement at peak (the 18th second after the injection of contrast agent), without a regular enhanced ring. (E) Contrast-enhanced ultrasound revealed heterogenous hypo-enhancement within the nodule at the 69th second after the injection of contrast agent, which indicates earlier wash-out than the surrounding thyroid parenchyma.

an integer-weighted score. Then, the risk of malignancy of each total point was estimated. Finally, we generated a point score that divided patients into different risk classes.

\section{Validation of the Predictive Model and Risk Score}

To validate the predictive model and the risk score, we used the data from our hospital that were not included in the derivation cohort between January 2020 and March 2021. 101 nodules in 100 consecutive patients, who met the same criteria in the derivation cohort, were included in the validation cohort.

\section{Statistical Analysis}

SPSS software (version 22.0, IBM Corporation, Armonk, NY, USA) and MedCalc (version 15.2, Mariakerke, Belgium) was used for statistical analysis. This derivation cohort was designed to have a minimum of 195 patients so as to achieve an expected sensitivity of 0.85 and a permissible error of 0.05 , at the 0.05 level of significance (two-tailed). The Shapiro-Wilk test (W test) was used to assess normal distributions of continuous variables. Normally distributed data were shown as mean \pm standard deviation and analyzed with the independent samples t-test, nonnormally distributed data were shown as median and interquartile range and analyzed with the Mann-Whitney U test. Data on categorical variables were shown as numbers and percentages. The chi-squared test or Fisher's exact test was conducted for categorical variables. Multivariate logistic regression analysis, including all of the variables from the univariate analysis that were associated with malignancy $(P<0.1)$, was used in the derivation sample to ascertain the independent risk factors and establish a risk predictive model. We used the HosmerLemeshow goodness-of-fit test and scatter diagram to assess model overfitting and calibration in the derivation cohort. Model's predictive discrimination was assessed using the $\mathrm{C}$ statistic (area under the receiver operating characteristic curve, AUC) in the derivation cohort.

To simplify this predictive model, we created a risk scoring system, which is based on the predicted probabilities of each total point. This risk scoring system divided nodules into low-risk (malignancy rate $<50 \%$ ) and high-risk (malignancy rate $\geq 50 \%$ ). To validate the model, we calculated the predicted probabilities 
for each nodule in the validation cohort using the predicted formula obtained in the derivation cohort. We assessed the discrimination by calculating the AUC in the validation cohort. The receiver operating characteristic (ROC) curves obtained by MedCalc were used to assess and compare the models' predictive performance. The sensitivity, specificity, positive predictive value (PPV), negative predictive value (NPV), and accuracy were calculated at the optimal cut-off points.

\section{RESULTS}

\section{Basic Characteristics}

Three hundred ten patients with 318 nodules were included in the present study. Table 1 summarizes the demographic and clinical characteristics of the patients with non-hypovascular thyroid nodules. The derivation cohort $(n=217)$ and validation cohort $(n=101)$ did not differ in their characteristics except to the location of thyroid nodules. In the derivation cohort, 88 nodules (40.6\%) were located in the upper of the thyroid lobe whereas in the validation cohort there were $23(22.8 \%)$ located in the upper $(P=0.008)$. Patients in the derivation cohort were slightly younger, with slightly larger nodules' size than in the validation cohort. Both cohorts showed the nodules were more common in females, without the background of Hashimoto's thyroiditis and multifocality.

\section{Pathological Diagnosis}

The derivation cohort consisted of 108 malignant nodules and 109 benign nodules. All malignant nodules (with or without FNA) were confirmed by surgery, which consisted of 74 papillary thyroid carcinomas (without subtype records), 20 classical variants of papillary thyroid carcinomas (4 with hyper-enhancement and 16 with iso-enhancement), 11 follicular variants of papillary thyroid

TABLE 1 | Basic characteristics in the derivation and validation cohorts.

\begin{tabular}{lccc}
\hline Parameters & Derivation (n = 217) & Validation (n = 101) & $\boldsymbol{P}$ \\
\hline Gender & & & $0.518^{*}$ \\
Female & $158(72.8)$ & $70(69.3)$ & \\
Male & $59(27.2)$ & $31(30.7)$ & \\
Age (y) & & & \\
Median & 48 & 51 & $0.203^{\#}$ \\
Interquartile & $37-57$ & $44-57$ & $0.619^{*}$ \\
$\geq 55$ & $67(30.9)$ & $34(33.7)$ & \\
$<55$ & $150(69.1)$ & $67(66.3)$ & $0.277^{\#}$ \\
Size (mm) & & & \\
Median & 16.0 & 15.0 & $0.008^{*}$ \\
interquartile & $12.0-21.0$ & $11.0-21.0$ & \\
Location & & & \\
Upper & $88(40.6)$ & $23(22.8)$ & \\
Middle & $43(19.8)$ & $25(24.8)$ & \\
Lower & $86(39.6)$ & $53(52.5)$ & \\
Multiple & & & \\
No & $119(54.8)$ & $54(53.5)$ & \\
Yes & $98(45.2)$ & $47(46.5)$ & \\
Hashimoto's thyroiditis & $178(82.0)$ & $83(82.2)$ & \\
No & $39(18.0)$ & $18(17.8)$ & \\
Yes & & & \\
\hline
\end{tabular}

*Determined with the $\chi^{2}$ test.

"Determined with the Mann-Whitney $U$ test. carcinomas (5 with hyper-enhancement and 6 with iso-enhancement), one oncocytic variant papillary thyroid carcinoma with hyper-enhancement, and three follicular thyroid carcinomas (all with hyper-enhancement). While among the 109 benign nodules, 40 were determined with FNA of Bethesda category II, and 69 were confirmed by surgery, which was made up of 36 nodular goiters, 15 follicular adenomas, 11 nodular goiters with local adenomatous hyperplasia, and seven Hashimoto's thyroiditis.

In the validation cohort, pathology demonstrated that 49 nodules $(48.5 \%)$ were malignant, and 52 nodules (51.5\%) were benign. All malignant nodules were confirmed by surgery, which consisted of 34 papillary thyroid carcinomas (without subtype records), 12 classical variants of papillary thyroid carcinomas, two follicular variants of papillary thyroid carcinomas, and one follicular thyroid carcinoma. Of the 52 benign nodules, 24 were determined with FNA, and 28 were confirmed by surgery, which included nine nodular goiters, five nodular goiters with adenomatous hyperplasia, 11 adenomas, and three nodular Hashimoto's thyroiditis.

\section{Univariate Analysis in the Derivation Cohort}

The conventional US and CEUS features and their assignments for the diagnosis of non-hypovascular benign and malignant thyroid nodules are shown in Table 2. There was a fair agreement for inter-observers in evaluating the ultrasonic features (kappa $=0.91)$. Shape, margin, and micro-calcification on the conventional US had statistical significance between the non-hypovascular benign and malignant nodules (all $P<0.05$ ). In benign non-hypovascular nodules, only 11 nodules $(10.1 \%)$ presented micro-calcification and 93 nodules (85.3\%) exhibited wider-than-tall. No significant difference was observed in the conventional US features of solid composition $(P=0.214)$, echogenicity $(P=0.683)$, and vascularity $(P=0.797)$. In comparison to benign non-hypovascular nodules, malignant non-hypovascular nodules had significant higher rate of heterogeneous enhancement $(91.7 \%$ vs. $69.7 \%, P<0.001$, Figures 4C-E), hyper-enhancement $(63.0 \%$ vs. $38.6 \%, P=$ 0.033 , Figures 3C, D and 4D), absence of regular ring enhancement ( $86.1 \%$ vs. $44.0 \%, P<0.001$, Figures 2D and 4D), ill-defined enhanced border (91.7\% vs. 69.7\%, $P<0.001$, Figure 2D), centripetal enhancement ( $24.1 \%$ vs. $6.4 \%, P<0.001$, Figures 2C and 4C), and earlier wash-out (66.1\% vs. $23.9 \%, P<0.001$, Figure 4E). Synchronous wash-in were more common in the benign nodules $(41.3 \%)$ than in the malignant nodules $(25.9 \%)$.

\section{Multivariate Analysis in the Derivation Cohort}

Multivariate logistic regression identified five independent risk factors for predicting malignancy of non-hypovascular thyroid nodules: presence of micro-calcification $\left(X_{5}\right)$, irregular margin $\left(X_{4}\right)$, earlier wash-out $\left(X_{13}\right)$, centripetal enhancement $\left(X_{11}\right)$, and absence of ring enhancement $\left(X_{9}\right)$ (Table 3 and Figures 1-4). Odds ratio $(\mathrm{OR})>1$ indicating risk factors and $\mathrm{OR}<1$ indicating protective factors. Micro-calcification had the largest OR value $(\mathrm{OR}=11.9,95 \% \mathrm{CI}: 4.463-30.728)$, with sensitivity, specificity, and accuracy of $59.3 \%, 89.9 \%$, and $74.7 \%$, respectively. Centripetal enhancement associated with malignancy had the highest specificity $(93.58 \%)$. Then a risk predictive model was 
TABLE 2 | Sonographic features of benign and malignant non-hypovascular thyroid nodules in the derivation cohort.

\begin{tabular}{|c|c|c|c|c|}
\hline Parameters & Assignment & $\begin{array}{l}\text { Benign } \\
(n=109)\end{array}$ & $\begin{array}{c}\text { Malignant } \\
(n=108)\end{array}$ & $P^{*}$ \\
\hline \multicolumn{5}{|c|}{ Conventional ultrasound features } \\
\hline Solid composition & $X_{1}$ & & & 0.214 \\
\hline No & 0 & $11(10.1)$ & $6(5.6)$ & \\
\hline Yes & 1 & 98(89.9) & 102(94.4) & \\
\hline Echogenicity & $x_{2}$ & & & 0.683 \\
\hline Hyper-/Isoechoic & 0 & 13(11.9) & 11(10.2) & \\
\hline (Markedly)Hypoechoic & 1 & 96(88.1) & 97(89.8) & \\
\hline Shape & $x_{3}$ & & & 0.005 \\
\hline Wider-than-tall & 0 & 93(85.3) & $75(69.4)$ & \\
\hline Taller-than-wide & 1 & $16(14.7)$ & 33(30.6) & \\
\hline Margin & $X_{4}$ & & & $<0.001$ \\
\hline Regular & 0 & $85(78.0)$ & $20(18.5)$ & \\
\hline Irregular & 1 & $24(22.0)$ & $88(81.5)$ & \\
\hline Micro-calcification & $X_{5}$ & & & $<0.001$ \\
\hline No & 0 & 98 (89.9) & $44(40.7)$ & \\
\hline Yes & 1 & $11(10.1)$ & $64(59.3)$ & \\
\hline Vascularity & $x_{6}$ & & & 0.797 \\
\hline None & 0 & $7(6.4)$ & $5(4.6)$ & \\
\hline Peripheral & 1 & $35(32.1)$ & $33(30.6)$ & \\
\hline Intranodular & 2 & $67(61.5)$ & $70(64.8)$ & \\
\hline \multicolumn{5}{|c|}{ Contrast-enhanced ultrasound features } \\
\hline Homogeneity & $x_{7}$ & & & $<0.001$ \\
\hline Homogeneous & 0 & 33 (30.3) & $9(8.3)$ & \\
\hline Heterogeneous & 1 & $76(69.7)$ & $99(91.7)$ & \\
\hline Enhanced intensity & $X_{8}$ & & & 0.033 \\
\hline Iso-enhancement & 0 & $56(51.4)$ & $40(37.0)$ & \\
\hline Hyper-enhancement & 1 & $53(48.6)$ & $68(63.0)$ & \\
\hline Ring enhancement & $X_{9}$ & & & $<0.001$ \\
\hline Present & 0 & $61(56.0)$ & $15(13.9)$ & \\
\hline Absent & 1 & $48(44.0)$ & $93(86.1)$ & \\
\hline Enhanced border & $x_{10}$ & & & $<0.001$ \\
\hline Well-defined & 0 & 93 (85.3) & $45(41.7)$ & \\
\hline III-defined & 1 & $16(14.7)$ & $63(58.3)$ & \\
\hline $\begin{array}{l}\text { Centripetal } \\
\text { enhancement }\end{array}$ & $X_{11}$ & & & $<0.001$ \\
\hline No & 0 & $\begin{array}{c}102 \\
(93.6)\end{array}$ & $82(75.9)$ & \\
\hline Yes & 1 & $7(6.4)$ & $26(24.1)$ & \\
\hline Wash-in & $X_{12}$ & & & 0.002 \\
\hline Synchronous & 0 & 45(41.3) & 28(25.9) & \\
\hline Later & 1 & $5(4.6)$ & 20(18.5) & \\
\hline Earlier & 2 & $59(54.1)$ & 60(55.6) & \\
\hline Wash-out & $X_{13}$ & & & $<0.001$ \\
\hline Synchronous & 0 & 41(37.6) & 16(14.8) & \\
\hline Later & 1 & 42(38.5) & $26(24.1)$ & \\
\hline Earlier & 2 & 26(23.9) & $66(61.1)$ & \\
\hline
\end{tabular}

*Determined with the $\chi^{2}$ test. established: $\operatorname{logit}(\mathrm{p})=-3.727+2.480 \times X_{5}+2.164 \times X_{4}+$ $1.482 \times X_{13}+1.254 \times X_{11}+1.203 \times X_{9}$. The model showed an AUC in diagnosing non-hypovascular malignant nodules was 0.921 (95\% CI, $0.876-0,953)$, with a sensitivity of $87.0 \%$, specificity of $86.2 \%$, PPV of $86.2 \%$, NPV of $87.0 \%$, and accuracy of $86.6 \%$, respectively (Table 4, Figure 5), which indicated the good discriminative ability of the model to distinguish non-hypovascular nodules who were malignant from those who were benign. The P-value of the HosmerLemeshow goodness-of-fit statistic with 8 degrees of freedom is 0.510 . The calibration intercept and slope were 0.0369 and 0.9966 in the derivation cohort (Supplementary Figure 1).

Then we compared the diagnostic performance of the predictive model (a combination of conventional US and CEUS) with conventional US and CEUS alone. The AUC of the predictive model $(0.921)$ was significantly higher than the conventional US $(0.892, P=0.0242)$ and CEUS (0.868, $P=0.0076)$ (Table 4 and Figure 5). In comparison to conventional US, CEUS had a higher specificity $(69.7 \%$ vs. $79.8 \%)$ and lower sensitivity (92.6\% vs. $81.5 \%)$ (Table 4$)$. But there was no difference $(P=0.3282)$ between the conventional US and CEUS in diagnosing non-hypovascular thyroid nodules.

\section{Development of Weighted Points and Establishment of the Risk Score}

The weighted points of each risk factor were displayed in Table 5. The total points of each non-hypovascular thyroid nodule were developed using the sum of weighted points of each predictor. The malignancy risk corresponded to each total points was shown in Table 6. According to the observed risk of malignancy of each total points, the nodules were divided into low-suspicious ( $0-3$ points; malignancy risk $<50 \%)$ and highsuspicious (4-7 points; malignancy risk $\geq 50 \%$ ) (Table 6). For non-hypovascular thyroid nodules $(\geq 10 \mathrm{~mm})$ with highsuspicious, FNA should be recommended.

\section{Validation of the Predictive Model and the Risk Score}

Variables used in the predictive model of the validation cohort were shown in Table 7 (more details in Supplementary Table 1). The diagnostic performance of the predictive model in the validation cohort was similar to that of the derivation cohort, with an AUC, sensitivity, specificity, PPV, and NPV in the validation cohort of 0.900 (95\% CI, 0.824-0.951), 85.7\%,

TABLE 3 | Multivariate analysis of the risk factors for malignancy in the derivation cohort.

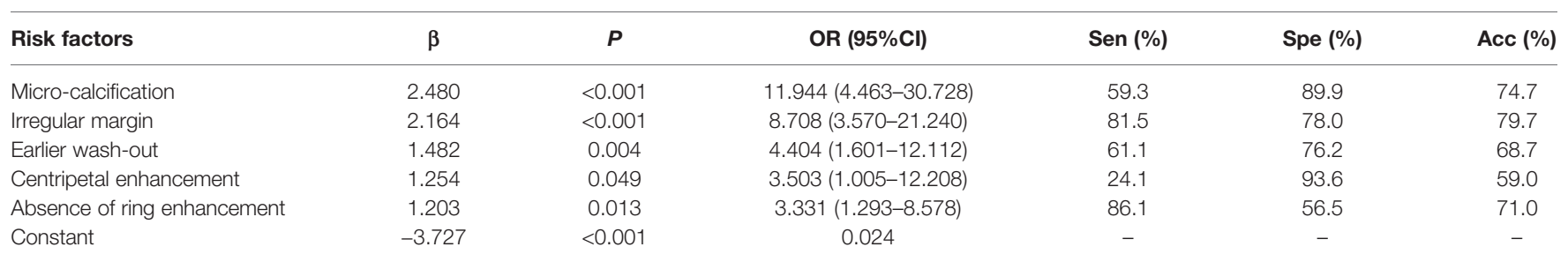

$\beta$, coefficient; OR, odds ration; Sen, sensitivity; Spe, specificity; Acc, accuracy. 
TABLE 4 | Diagnostic performance of conventional US, CEUS, and predictive model in the derivation cohort.

\begin{tabular}{|c|c|c|c|c|c|c|c|}
\hline Methods & Sen $(\%)$ & Spe (\%) & PPV (\%) & NPV (\%) & Acc (\%) & AUC & $P$ \\
\hline CEUS & 81.5 & 79.8 & 80.2 & 82.1 & 80.6 & $0.868(0.815-0.910)$ & $0.0242^{\#}$ \\
\hline Predictive model & 87.0 & 86.2 & 86.2 & 87.0 & 86.6 & $0.921(0.876-0.953)$ & $0.0076^{\wedge}$ \\
\hline
\end{tabular}

Sen, sensitivity; Spe, specificity; PPV, positive predictive value; NPV, negative predictive value; Acc, accuracy; AUC, area under the curve.

${ }^{*}$ Conventional US vs. CEUS.

"Predictive model vs. Conventional US.

${ }^{\wedge}$ Predictive model vs. CEUS.

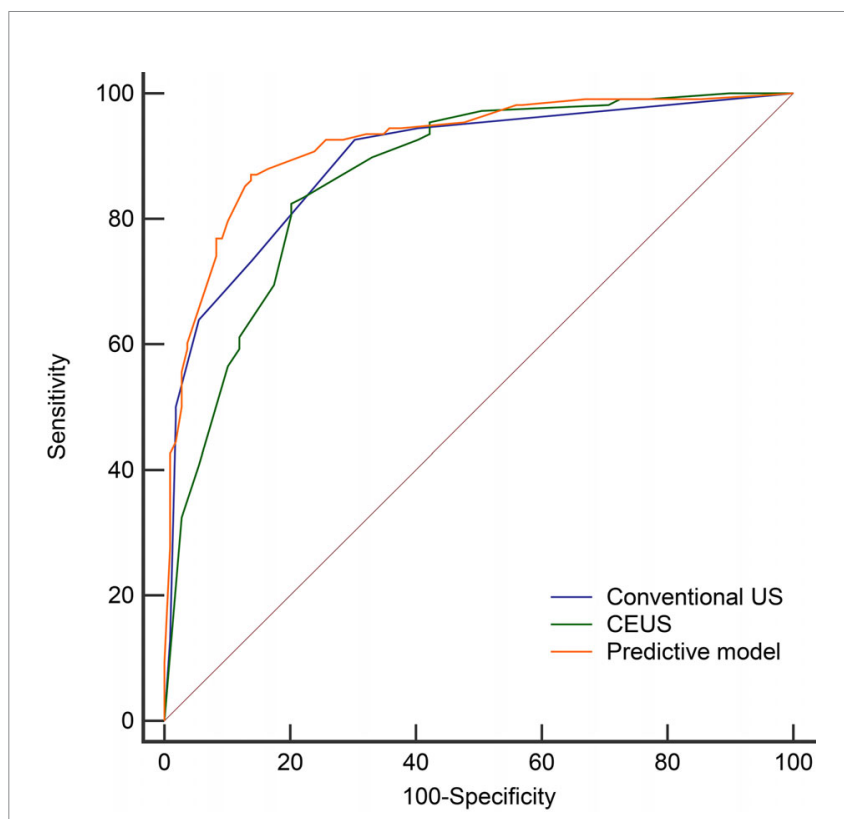

FIGURE 5 | The Receiver operating characteristic curves of the conventional


the derivation cohort.

$88.5 \%, 87.5 \%$, and $86.8 \%$, respectively (Figure 6). The performance of the risk score in the validation cohort was summarized in Table 8. High accuracy $(87.1 \%)$ can be observed using the risk score in the validation cohort.

\section{Comparing Two Methods in Screening Non-Hypovascular Thyroid Nodules for FNA in the Derivation Cohort}

According to the recommended criteria of ACR TI-RADS (18), there were 170 nodules $(78.3 \%, 170 / 217)$ suitable for FNA. While using the risk stratification method in this study, only 92 nodules (42.4\%, 92/217) were recommended for FNA (Table 9). Comparing ACR TI-RADS in screening suitable nodules for FNA, the risk stratification method could avoid 30.8\% (69/1709/92) benign nodules for FNA.

\section{DISCUSSION}

The early detection, accurate diagnosis of the benignity and malignancy of thyroid nodules is of considerable significance for
TABLE 5 | Development of weighted points for risk factors in the derivation cohort.

\begin{tabular}{|c|c|c|c|c|c|}
\hline Risk factors & Categories & $\begin{array}{l}\text { Reference } \\
\text { value }\left(X_{\mathrm{ij}}\right)\end{array}$ & $\beta_{\mathrm{i}}$ & $\begin{array}{c}\beta_{\mathrm{i}} \\
\left(X_{\mathrm{ij}}-X_{\mathrm{iREF}}\right)\end{array}$ & Points \\
\hline \multirow{3}{*}{$\begin{array}{l}\text { Micro- } \\
\text { calcification }\end{array}$} & & & 2.480 & & \\
\hline & No & $0=X_{5 R E F}$ & & 0 & 0 \\
\hline & Yes & 1 & & 2.480 & 2 \\
\hline \multirow[t]{3}{*}{ Margin } & & & 2.164 & & \\
\hline & Regular & $0=X_{4 \mathrm{REF}}$ & & 0 & 0 \\
\hline & Irregular & 1 & & 2.164 & 2 \\
\hline \multirow[t]{3}{*}{ Earlier wash-out } & & & 1.482 & & \\
\hline & No & $0=X_{13 R E F}$ & & 0 & 0 \\
\hline & Yes & 1 & & 1.482 & 1 \\
\hline \multirow{3}{*}{$\begin{array}{l}\text { Centripetal } \\
\text { enhancement }\end{array}$} & & & 1.254 & & \\
\hline & No & $0=X_{11 \mathrm{REF}}$ & & 0 & 0 \\
\hline & Yes & 1 & & 1.254 & 1 \\
\hline \multirow{3}{*}{$\begin{array}{l}\text { Ring } \\
\text { enhancement }\end{array}$} & & & 1.203 & & \\
\hline & presence & $0=X_{\text {gREF }}$ & & 0 & 0 \\
\hline & absence & 1 & & 1.203 & 1 \\
\hline
\end{tabular}

Scores $=\beta_{i}\left(X_{i j}-X_{\text {iREF }}\right) / \beta_{8}$.

TABLE 6 | Risk stratification according to the malignancy risk of each total points.

\begin{tabular}{lcc}
\hline Risk stratification method & Total points & Risk of malignancy (\%) \\
\hline Low-suspicious & 0 & 2.4 \\
& 1 & 7.4 \\
& 2 & 21.1 \\
High-suspicious & 3 & 47.1 \\
& 4 & 74.7 \\
& 5 & 90.8 \\
& 6 & 97.0 \\
7 & 99.1 \\
\hline
\end{tabular}

the clinical management of thyroid nodules. Ultrasound is the preferred imaging method for diagnosing thyroid nodules. The clinical approach to thyroid nodules has recently changed to reduce the number of unnecessary biopsies while improving the diagnostic accuracy of the sonographic appearance in most detected thyroid nodules $(23,24)$. Therefore, improving the diagnostic accuracy of sonographic appearance while reducing the unnecessary FNA of thyroid nodules is quite crucial.

To identify the most clinically significant malignancies while reducing the number of FNA performed on benign nodules, in 2017, the ACR TI-RADS Committee presented their system for risk 
stratification to provide guidance regarding the management of thyroid nodules on the basis of their conventional US appearance (18). By using the ACR TI-RADS to estimate the malignancy risk of thyroid nodules, a low specificity was obtained in our medical

TABLE 7 | Features of risk factors of non-hypovascular thyroid nodules in the validation cohort.

\begin{tabular}{lccc}
\hline Parameters & Assignment & Benign (n=52) & Malignant (n=49) \\
\hline Conventional ultrasound features & & & \\
Margin & $X_{4}$ & & \\
$\quad$ Regular & 0 & $39(75.0)$ & $8(16.3)$ \\
Irregular & 1 & $13(25.0)$ & $41(83.7)$ \\
Micro-calcification & $X_{5}$ & & \\
No & 0 & $41(78.8)$ & $17(34.7)$ \\
Yes & 1 & $11(21.2)$ & $32(65.3)$ \\
Contrast-enhanced ultrasound features & & \\
Ring enhancement & $X_{9}$ & & \\
Present & 0 & $30(57.7)$ & $6(12.2)$ \\
Absent & 1 & $22(42.3)$ & $43(87.8)$ \\
Centripetal enhancement & $X_{11}$ & & \\
No & 0 & $47(90.4)$ & $30(61.2)$ \\
Yes & 1 & $5(9.6)$ & $19(38.8)$ \\
Wash-out & $X_{13}$ & & \\
Synchronous & 0 & $12(25.0)$ & $13(26.5)$ \\
Later & 1 & $10(19.2)$ & $6(12.2)$ \\
Earlier & 2 & $29(55.8)$ & $30(61.2)$ \\
\end{tabular}

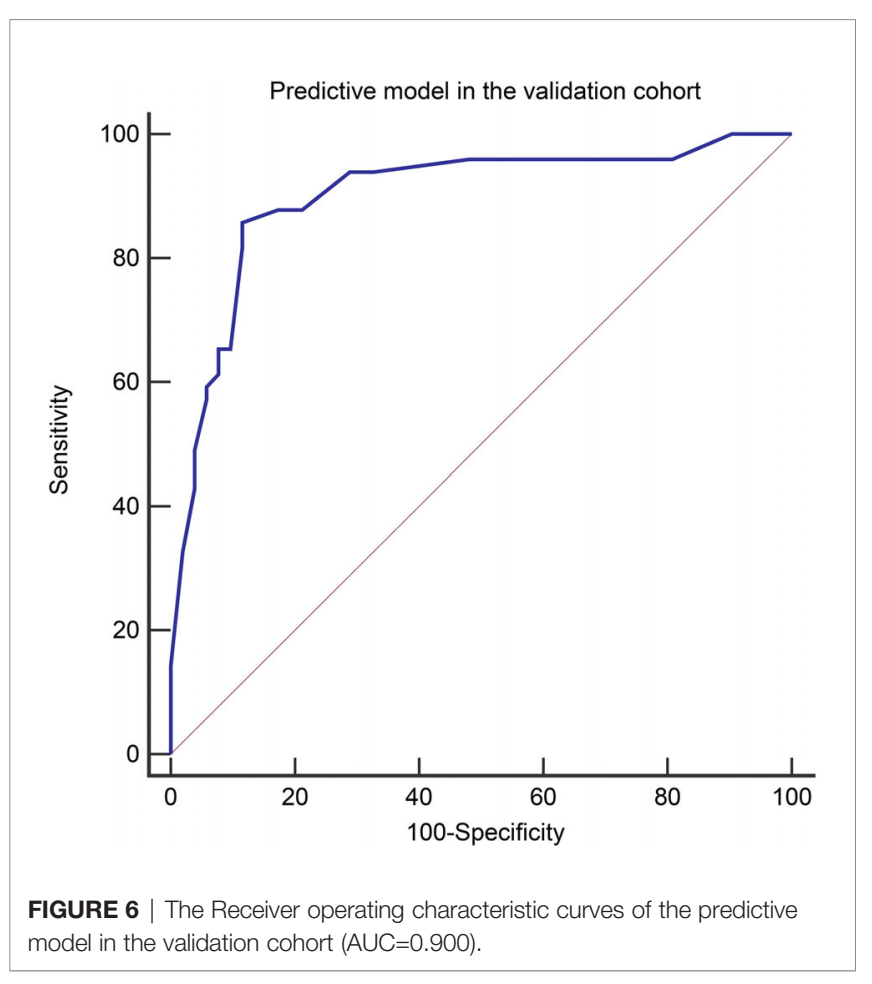

center, especially in nodules with ACR TI-RADS category 4. This may be due to some benign nodules, such as thyroid adenoma, chronic lymphocytic thyroiditis, and even nodular goiter (for example, mummy nodule), may present as hypoechoic with an irregular margin (25). Therefore, ACR TI-RADS has some limitations in the risk stratification of thyroid nodules. Other ultrasound techniques, such as CEUS and elastography, are needed.

CEUS is considered to be an effective technique to evaluate micro-vascularization, which is much important because angiogenesis is the basis for neoplastic growth (5). Recent metaanalyses showed both the sensitivity and specificity of CEUS in diagnosing thyroid nodules were more than $82 \%(26,27)$. Among the CEUS features of thyroid nodules, hypo-enhancement has been confirmed to be associated with malignancy by numerous studies $(5,28-32)$. However, not all malignant nodules appear to be hypoenhancement on CEUS in clinical practice. Some researchers reported improved TI-RADS combined with CEUS could improve the diagnostic accuracy of thyroid nodules and reduce the number of FNA $(15,25)$. But they included all classifications of thyroid nodules and did not further subdivide the intensity of enhancement. In this study, we focused on ACR-TI RDS category 4 and 5 nodules only with iso-/hyper-enhancement on CEUS. The malignancy rate in the derivation cohort was only $49.8 \%(108 / 217)$. All of these non-hypovascular nodules were subjected to FNA and/ or surgery, which may already bring the overdiagnosis and treatment. But this condition is not uncommon in many cities in China due to the patient's anxiety and doctors' worry about the missed diagnosis. To minimize the costs and maximize the benefits of FNA, and to prevent unnecessary diagnostic surgery (33), this study investigated the value of CEUS in the diagnosis and risk stratification of non-hypovascular thyroid nodules $(\geq 10 \mathrm{~mm})$. The results showed that CEUS is comparable to conventional US in distinguishing non-hypovascular thyroid nodules but a combination of conventional US and CEUS has superior performance than the single method. The presence of microcalcification, irregular margin, earlier wash-out, centripetal enhancement, and absence of ring enhancement were independent risk indicators. By weighting these risk indicators, we developed a simple risk score and divided the non-hypovascular

TABLE 9 | Comparing two methods in screening non-hypovascular thyroid nodules for FNA.

\begin{tabular}{lcccc}
\hline & $\begin{array}{c}\text { Nodules suitable for } \\
\text { FNA }\end{array}$ & \multicolumn{2}{c}{ Pathology } & \multirow{2}{*}{$\begin{array}{c}\text { Malignancy } \\
\text { rate }\end{array}$} \\
\cline { 3 - 3 } & 170 & 69 & 101 & $59.4 \%$ \\
$\begin{array}{l}\text { ACR TI- } \\
\begin{array}{l}\text { RADS } \\
\text { Risk score }\end{array}\end{array}$ & 92 & 9 & 83 & $90.2 \%$ \\
\hline
\end{tabular}

FNA, fine-needle aspiration.

TABLE 8 | Validation of the risk score.

\begin{tabular}{|c|c|c|c|c|c|c|c|}
\hline Risk score & Benign & Malignant & Sensitivity & Specificity & PPV & NPV & Accuracy \\
\hline Low-suspicious & 46 & 7 & $85.7 \%$ & $88.5 \%$ & $87.5 \%$ & $86.8 \%$ & $87.1 \%$ \\
\hline High-suspicious & 6 & 42 & & & & & \\
\hline
\end{tabular}

PPV, positive predictive value; NPV, negative predictive value. 
nodules into two categories: low-suspicious (0-3 points) and high-suspicious (4-7 points). By using the risk score, $30.8 \%$ of benign nodules could avoid FNA.

The well-accepted conventional US features associated with malignancy include solid composition, (markedly) hypoechoic, taller-than-wide, irregular margin, and microcalcification $(18,34)$. In the present study, we not only analyzed the above features but also the vascularity by color Doppler, as we focused on the nonhypovascular thyroid nodules. The results showed that microcalcification and irregular margin were correlated with malignancy for non-hypovascular thyroid nodules, with sensitivity and specificity of $59.3 \%$ and $89.9 \%, 81.5 \%$ and $78.0 \%$, respectively. This indicated no single feature was sensitive and specific enough in the diagnosis of non-hypovascular thyroid nodules. Although a taller-than-wide shape was more frequently found in malignant nodules, it was not specific for nonhypovascular nodules. Solid composition, (markedly) hypoechoic, and vascularity were not independent risk factors in the current study. This can be explained by the fact that $92.2 \%(200 / 217)$ of the non-hypovascular thyroid nodules were solid composition and $88.9 \%$ (193/217) with (markedly) hypo-echogenicity in the derivation cohort. The vascularity assessed by Doppler ultrasound in the diagnosis of thyroid nodules is controversial. Some authors claim that intranodular vascularity is associated with malignancy for thyroid nodules $(35,36)$, while others demonstrate that it is not helpful to predict malignancy $(19,37)$. In our study, the results showed the vascularity was less helpful in differentiating nonhypovascular thyroid nodules. This may be to the low sensitivity of color Doppler in detecting vascularity within thyroid nodules.

CEUS can make up for the low sensitivity of color Doppler in detecting vascularity within tumors. Though CEUS can be analyzed qualitatively or quantitatively, there is no unified CEUS terminology for qualitative or quantitative analysis of thyroid nodules at present. And no single CEUS features seem to be sufficiently sensitive or specific to distinguish between benign and malignant thyroid nodules. A previous meta-analysis found that qualitative evaluation acquired better sensitivity and specificity than quantitative evaluation in differentiating thyroid nodules (38). In this study, we analyzed seven qualitative CEUS variables (homogeneity, enhanced intensity, ring enhancement, enhanced border, centripetal enhancement, relative wash-in time, and relative wash-out time). The results of the univariate analysis in the derivation cohort showed that all variables were significantly different between benign and malignant non-hypovascular nodules. We observed that non-hypovascular malignant thyroid nodules on CEUS could be heterogeneous, hyper-enhancement, absence of ring enhancement, ill-defined enhanced border, centripetal enhancement, earlier wash-in, and earlier wash-out. These findings seemed to be not exactly the same as Wu et. al's (39), which concluded later arrival, centripetal mode of entrance, hypo-enhancement, heterogeneous enhancement, and earlier wash-out were CEUS diagnostic criteria of malignant nodules. This may be due to the select difference as we exclude nodules with hypo-enhancement and non-enhancement. Different enhancement modes are related to the corresponding pathologic mechanisms (40). Numerous studies (11, 32, 41-44) have demonstrated hypo-enhancement and heterogeneous enhancement are major features of malignancy thyroid nodules. Possible explanations were necrosis, calcification, fibrosis, and embolus formation within the tumor (43). The reason for those malignant nodules appeared iso-/hyper-enhancement on CEUS is unclear yet. In the derivation cohort, we found 3(100\%) follicular thyroid carcinomas, $5(45.4 \%, 5 / 11)$ follicular variants of papillary thyroid carcinomas, and $4(20 \%, 4 / 20)$ classical variants of papillary thyroid carcinomas all with hyper-enhancement. As follicular tumors are usually hyper-vascular, it is possible to speculate that non-hypovascular malignant nodules with hyperenhancement on CEUS may be more correlated with follicular thyroid carcinoma and follicular variants of papillary thyroid carcinoma. Unfortunately, there was no pathological record on the variants of 74 papillary thyroid carcinoma in this study. We will further study them shortly.

In general, tumor neovascularization is relatively dense in the marginal zone and sparse in the center, which may lead to centripetal enhancement and heterogeneous enhancement in the malignant nodules (39). In addition, the immature new blood vessels usually have a low resistance index and may exist arteriovenous fistula, both earlier wash-in and earlier wash-out might be present during the CEUS process. A thin and regular ring enhancement is a feature of benign nodules, especially for follicular adenoma. When the malignant nodules grow unevenly and invade the surrounding normal parenchyma, the ring may be incomplete or blurred, thus may resulting in absence of ring enhancement and ill-defined enhanced border. In this study, the results showed that earlier wash-out, centripetal enhancement, and absence of ring enhancement were independent CEUS indicators associated with malignancy for non-hypovascular nodules. Centripetal enhancement had the highest specificity (93.6\%) and the absence of ring enhancement had the highest sensitivity $(86.1 \%)$ in diagnosing non-hypovascular malignant thyroid nodules, which could attribute to an increase in accuracy of distinguishing benign nodules and prevent patients with a further invasive procedure. Similar results were also reported by other researchers $(11,30,45)$.

A prior study reported the diagnostic accuracy of contrastenhanced ultrasound (CEUS) for distinguishing malignant thyroid nodules from benign thyroid nodules remains controversial (26). In our study, CEUS is comparable to conventional US in distinguishing non-hypovascular thyroid nodules. And CEUS had high specificity than conventional US (79.8\% vs. 69.7\%) in differential diagnosing non-hypovascular nodules, which indicated a better capability of identifying benign nodules and avoiding unessential FNA. By a combination of conventional US and CEUS, Ma et al. (46) reported that the sensitivity can up to $89.4 \%$ (84/94) and specificity of $93.6 \%(73 /$ 78). $\mathrm{Xu}$ et al. (47) demonstrated that the sensitivity, specificity, and AUC of the combined method were $85.7 \%, 83.3 \%$, and 0.867 , respectively. Our study also confirmed the good diagnostic efficacy of a combination for diagnosing non-hypovascular thyroid nodules, with an AUC, sensitivity, and specificity of $0.921,87.0 \%$, and $86.2 \%$ in the derivation cohort and 0.900 , $85.7 \%$, and $88.5 \%$ in the external validation cohort, respectively. The AUC of the combined method in the derivation cohort in 
this study was significantly higher than the conventional US $(Z=2.255, P=0.0242)$ and CEUS $(Z=2.671, P=0.0076)$ alone

(Figure 5), which was in line with published articles $(14,15)$.

Recently, some studies have also reported prediction models for differentiating benign and malignant thyroid nodules (22, 42, 4850). However, few of them weighted for the risk factors and it was inconvenient to calculate the risk of malignancy clinically. In this study, we weighed for these risk factors and developed a risk score, which could divide the non-hypovascular nodules into different risk classes with a simpler method. By using this risk score in the validation cohort, the risk score exhibited the good ability of risk stratification, with a sensitivity of $85.7 \%$, sensitivity of $88.5 \%$, and accuracy of $87.1 \%$, respectively. Moreover, by comparing ACR TIRADS and risk score in screening suitable nodules for FNA, we found the risk score could avoid $30.8 \%$ benign nodules for FNA. This meant the risk score had better performance than ACR TIRADS in the clinical management of non-hypovascular thyroid nodules. The reduced number of FNA mainly comes from the ACR TI-RADS category 4. Therefore, the risk score can help us distinguish non-hypovascular thyroid nodules (especially for ACR TI-RADS category 4) more simply, avoid unnecessary FNA, and improve the malignancy rate of FNA.

At present, few reports describe the risk stratification of nonhypovascular thyroid nodules using conventional US features and CEUS features. This study provides a simple and practical risk score to estimate the malignancy risk level of non-hypovascular thyroid nodules. Using this risk score, radiologists could diagnose and stratify the non-hypovascular thyroid nodules more conveniently and accurately, helping screening necessary nodules for further FNA. However, this study has several limitations. First, a selection bias may be present that we excluded nodules with no-enhancement and hypo-enhancement. Second, the malignant thyroid nodules were mainly papillary thyroid carcinoma, while other histological types were rare and the variants of 74 papillary thyroid carcinoma were unclear. Third, as the present study was a single-center study with small sample size, additional multi-center studies, and larger sample sizes are needed in the future.

\section{CONCLUSIONS}

In conclusion, we developed a risk score based on significant conventional US features and CEUS features to differential diagnose and stratify non-hypovascular thyroid nodules. The risk score was validated externally and prove to be reproducible with good performance. CEUS is comparable to conventional US in distinguishing non-hypovascular thyroid nodules, but a combination of them has the potential to improve the

\section{REFERENCES}

1. Moon WJ, Baek JH, Jung SL, Kim DW, Kim EK, Kim JY, et al. Ultrasonography and the Ultrasound-Based Management of Thyroid Nodules: Consensus Statement and Recommendations. Kor J Radiol (2011) 12:1-14. doi: 10.3348/kjr.2011.12.1.1

2. Smith-Bindman R, Lebda P, Feldstein VA, Sellami D, Goldstein RB, Brasic N, et al. Risk of Thyroid Cancer Based on Thyroid Ultrasound Imaging individualized management of non-hypovascular thyroid nodules, avoiding unnecessary FNA for benign nodules.

\section{DATA AVAILABILITY STATEMENT}

The original contributions presented in the study are included in the article/Supplementary Material. Further inquiries can be directed to the corresponding author.

\section{ETHICS STATEMENT}

The studies involving human participants were reviewed and approved by Ethics Committee of Lanzhou University Second Hospital. The patients/participants provided their written informed consent to participate in this study.

\section{AUTHOR CONTRIBUTIONS}

Conception and design of the study: YW and FN. Ultrasound data acquisition: YW and GW. Clinical and pathological data collection: GW and QN. Analysis and interpretation of data: TD and TL. Drafting the manuscript: YW. Revising and final approval of the version to be published: TD and FN. All authors contributed to the article and approved the submitted version.

\section{FUNDING}

This research was supported by the Key Talent Projects of Gansu Province in 2019 (Grant number: 2019RCXM021).

\section{ACKNOWLEDGMENTS}

The authors thank all team members and colleagues in the Medical Center of Ultrasound, Lanzhou University Second Hospital for their helpful cooperation and all the study participants for their patience and support.

\section{SUPPLEMENTARY MATERIAL}

The Supplementary Material for this article can be found online at: https://www.frontiersin.org/articles/10.3389/fonc.2021. 662273/full\#supplementary-material

Characteristics: Results of a Population-Based Study. JAMA Intern Med (2013) 173:1788-96. doi: 10.1001/jamainternmed.2013.9245

3. Tan GH, Gharib H. Thyroid Incidentalomas: Management Approaches to Nonpalpable Nodules Discovered Incidentally on Thyroid Imaging. Ann Intern Med (1997) 126:226-31. doi: 10.7326/0003-4819-126-3-19970201000009

4. Guth S, Theune U, Aberle J, Galach A, Bamberger CM. Very High Prevalence of Thyroid Nodules Detected by High Frequency $(13 \mathrm{MHz})$ Ultrasound 
Examination. Eur J Clin Invest (2009) 39:699-706. doi: 10.1111/j.13652362.2009.02162.x

5. Sidhu PS, Cantisani V, Dietrich CF, Gilja OH, Saftoiu A, Bartels E, et al. The EFSUMB Guidelines and Recommendations for the Clinical Practice of Contrast-Enhanced Ultrasound (CEUS) in Non-Hepatic Applications: Update 2017 (Long Version). Ultraschall Med (2018) 39:e2-e44. doi: 10.1055/a-0586-1107

6. Bongiovanni M, Spitale A, Faquin WC, Mazzucchelli L, Baloch ZW. The Bethesda System for Reporting Thyroid Cytopathology: A Meta-Analysis. Acta Cytol (2012) 56:333-9. doi: 10.1159/000339959

7. Cibas ES, Ali SZ. The 2017 Bethesda System for Reporting Thyroid Cytopathology. Thyroid (2017) 27:1341-6. doi: 10.1089/thy.2017.0500

8. Durante C, Grani G, Lamartina L, Filetti S, Mandel SJ, Cooper DS. The Diagnosis and Management of Thyroid Nodules: A Review. JAMA (2018) 319:914-24. doi: 10.1001/jama.2018.0898

9. Albrecht T, Blomley M, Bolondi L, Claudon M, Correas JM, Cosgrove D, et al. Guidelines for the Use of Contrast Agents in Ultrasound. January 2004. Ultraschall Med (2004) 25:249-56. doi: 10.1055/s-2004-813245

10. Dietrich CF, Nolsoe CP, Barr RG, Berzigotti A, Burns PN, Cantisani V, et al. Guidelines and Good Clinical Practice Recommendations for Contrast Enhanced Ultrasound (CEUS) in the Liver - Update 2020 - WFUMB in Cooperation With EFSUMB, AFSUMB, AIUM, and FLAUS. Ultraschall Med (2020) 41:562-85. doi: 10.1055/a-1177-0530

11. Zhang B, Jiang YX, Liu JB, Yang M, Dai Q, Zhu QL, et al. Utility of ContrastEnhanced Ultrasound for Evaluation of Thyroid Nodules. Thyroid (2010) 20:51-7. doi: 10.1089/thy.2009.0045

12. Zhang Y, Zhang MB, Luo YK, Li J, Wang ZL, Tang J. The Value of Peripheral Enhancement Pattern for Diagnosing Thyroid Cancer Using ContrastEnhanced Ultrasound. Int J Endocrinol (2018) 2018:1625958. doi: 10.1155/ 2018/1625958

13. Huang R, Jiang $\mathrm{L}, \mathrm{Xu} \mathrm{Y}$, Gong $\mathrm{Y}$, Ran $\mathrm{H}$, Wang $\mathrm{Z}$, et al. Comparative Diagnostic Accuracy of Contrast-Enhanced Ultrasound and Shear Wave Elastography in Differentiating Benign and Malignant Lesions: A Network Meta-Analysis. Front Oncol (2019) 9:102. doi: 10.3389/fonc.2019.00102

14. Zhao H, Liu X, Lei B, Cheng P, Li J, Wu Y, et al. Diagnostic Performance of Thyroid Imaging Reporting and Data System (TI-RADS) Alone and in Combination With Contrast-Enhanced Ultrasonography for the Characterization of Thyroid Nodules. Clin Hemorheol Microcirc (2019) 72:95-106. doi: $10.3233 / \mathrm{CH}-180457$

15. Zhang Y, Zhou P, Tian SM, Zhao YF, Li JL, Li L. Usefulness of Combined Use of Contrast-Enhanced Ultrasound and TI-RADS Classification for the Differentiation of Benign From Malignant Lesions of Thyroid Nodules. Eur Radiol (2017) 27:1527-36. doi: 10.1007/s00330-016-4508-y

16. Zhang L, Gu J, Zhao Y, Zhu M, Wei J, Zhang B. The Role of Multimodal Ultrasonic Flow Imaging in Thyroid Imaging Reporting and Data System (TiRADS) 4 Nodules. Gland Surg (2020) 9:1469-77. doi: 10.21037/gs-20-641

17. Zhou Q, Xu YB, Jiang J, Ma WQ, Wang H, Li M, et al. [Differential Diagnostic Value of Contrast-Enhanced Ultrasound in Calcified Thyroid Nodules]. Zhonghua Er Bi Yan Hou Tou Jing Wai Ke Za Zhi (2013) 48:726-9.

18. Tessler FN, Middleton WD, Grant EG, Hoang JK, Berland LL, Teefey SA, et al. Acr Thyroid Imaging, Reporting and Data System (Ti-Rads): White Paper of the ACR Ti-RADS Committee. J Am Coll Radiol (2017) 14:587-95. doi: 10.1016/j.jacr.2017.01.046

19. Moon HJ, Kwak JY, Kim MJ, Son EJ, Kim EK. Can Vascularity at Power Doppler US Help Predict Thyroid Malignancy? Radiology (2010) 255:260-9. doi: 10.1148/radiol.09091284

20. Anderson RP, Jin R, Grunkemeier GL. Understanding Logistic Regression Analysis in Clinical Reports: An Introduction. Ann Thorac Surg (2003) 75:753-7. doi: 10.1016/s0003-4975(02)04683-0

21. Sperandei S. Understanding Logistic Regression Analysis. Biochem Med (Zagreb) (2014) 24:12-8. doi: 10.11613/BM.2014.003

22. Pang T, Huang L, Deng Y, Wang T, Chen S, Gong X, et al. Logistic Regression Analysis of Conventional Ultrasonography, Strain Elastosonography, and Contrast-Enhanced Ultrasound Characteristics for the Differentiation of Benign and Malignant Thyroid Nodules. PloS One (2017) 12:e0188987. doi: 10.1371/journal.pone.0188987

23. Castellana M, Piccardo A, Virili C, Scappaticcio L, Grani G, Durante C, et al. Can Ultrasound Systems for Risk Stratification of Thyroid Nodules Identify
Follicular Carcinoma? Cancer Cytopathol (2020) 128:250-9. doi: 10.1002/ cncy.22235

24. Grani G, Lamartina L, Ascoli V, Bosco D, Biffoni M, Giacomelli L, et al. Reducing the Number of Unnecessary Thyroid Biopsies While Improving Diagnostic Accuracy: Toward the "Right" Tirads. J Clin Endocrinol Metab (2019) 104:95-102. doi: 10.1210/jc.2018-01674

25. Jin ZQ, Yu HZ, Mo CJ, Su RQ. Clinical Study of the Prediction of Malignancy in Thyroid Nodules: Modified Score Versus 2017 American College of Radiology's Thyroid Imaging Reporting and Data System Ultrasound Lexicon. Ultrasound Med Biol (2019) 45:1627-37. doi: 10.1016/ j.ultrasmedbio.2019.03.014

26. Zhang J, Zhang X, Meng Y, Chen Y. Contrast-Enhanced Ultrasound for the Differential Diagnosis of Thyroid Nodules: An Updated Meta-Analysis With Comprehensive Heterogeneity Analysis. PloS One (2020) 15:e231775. doi: 10.1371/journal.pone.0231775

27. Trimboli P, Castellana M, Virili C, Havre RF, Bini F, Marinozzi F, et al. Performance of Contrast-Enhanced Ultrasound (CEUS) in Assessing Thyroid Nodules: A Systematic Review and Meta-Analysis Using Histological Standard of Reference. Radiol Med (2020) 125:406-15. doi: 10.1007/s11547019-01129-2

28. Zhang Y, Luo YK, Zhang MB, Li J, Li J, Tang J. Diagnostic Accuracy of Contrast-Enhanced Ultrasound Enhancement Patterns for Thyroid Nodules. Med Sci Monit (2016) 22:4755-64. doi: 10.12659/msm.899834

29. Deng J, Zhou P, Tian SM, Zhang L, Li JL, Qian Y. Comparison of Diagnostic Efficacy of Contrast-Enhanced Ultrasound, Acoustic Radiation Force Impulse Imaging, and Their Combined Use in Differentiating Focal Solid Thyroid Nodules. PloS One (2014) 9:e90674. doi: 10.1371/journal.pone.0090674

30. Yuan Z, Quan J, Yunxiao Z, Jian C, Zhu H. Contrast-Enhanced Ultrasound in the Diagnosis of Solitary Thyroid Nodules. J Cancer Res Ther (2015) 11:41-5. doi: 10.4103/0973-1482.147382

31. Li F, Luo H. Comparative Study of Thyroid Puncture Biopsy Guided by Contrast-Enhanced Ultrasonography and Conventional Ultrasound. Exp Ther Med (2013) 5:1381-4. doi: 10.3892/etm.2013.1016

32. Jiang J, Shang X, Wang H, Xu YB, Gao Y, Zhou Q. Diagnostic Value of Contrast-Enhanced Ultrasound in Thyroid Nodules With Calcification. Kaohsiung J Med Sci (2015) 31:138-44. doi: 10.1016/j.kjms.2014.12.001

33. Zhang Y, Meng F, Hong L, Chu L. A Risk Score Model for Evaluation and Management of Patients With Thyroid Nodules. Horm Metab Res (2018) 50:543-50. doi: 10.1055/a-0630-5239

34. Kwak JY, Han KH, Yoon JH, Moon HJ, Son EJ, Park SH, et al. Thyroid Imaging Reporting and Data System for US Features of Nodules: A Step in Establishing Better Stratification of Cancer Risk. Radiology (2011) 260:892-9. doi: 10.1148/radiol.11110206

35. Baig FN, Lunenburg J, Liu SYW, Yip SP, Law HKW, Ying M. ComputerAided Assessment of Regional Vascularity of Thyroid Nodules for Prediction of Malignancy. Sci Rep (2017) 7:14350. doi: 10.1038/s41598-017-14432-7

36. Varverakis E, Neonakis E, Tzardi M, Chrysos E. Role of Color Doppler Ultrasonography in the Preoperative Management of Cold Thyroid Nodules. Hormones (Athens) (2007) 6:44-51.

37. Yoon JH, Kim EK, Kwak JY, Park VY, Moon HJ. Application of Various Additional Imaging Techniques for Thyroid Ultrasound: Direct Comparison of Combined Various Elastography and Doppler Parameters to Gray-Scale Ultrasound in Differential Diagnosis of Thyroid Nodules. Ultrasound Med Biol (2018) 44:1679-86. doi: 10.1016/j.ultrasmedbio.2018.04.006

38. Yu D, Han Y, Chen T. Contrast-Enhanced Ultrasound for Differentiation of Benign and Malignant Thyroid Lesions: Meta-Analysis. Otolaryngol Head Neck Surg (2014) 151:909-15. doi: 10.1177/0194599814555838

39. Wu Q, Wang Y, Li Y, Hu B, He ZY. Diagnostic Value of Contrast-Enhanced Ultrasound in Solid Thyroid Nodules With and Without Enhancement. Endocrine (2016) 53:480-8. doi: 10.1007/s12020-015-0850-0

40. Luo W, Numata K, Morimoto M, Kondo M, Takebayashi S, Okada M, et al. Focal Liver Tumors: Characterization With 3D Perflubutane Microbubble Contrast Agent-Enhanced US Versus 3D Contrast-Enhanced Multidetector CT. Radiology (2009) 251:287-95. doi: 10.1148/radiol.2511081324

41. Schleder S, Janke M, Agha A, Schacherer D, Hornung M, Schlitt HJ, et al. Preoperative Differentiation of Thyroid Adenomas and Thyroid Carcinomas Using High Resolution Contrast-Enhanced Ultrasound (CEUS). Clin Hemorheol Microcirc (2015) 61:13-22. doi: 10.3233/ch-141848 
42. Zhao RN, Zhang B, Yang X, Jiang YX, Lai XJ, Zhang XY. Logistic Regression Analysis of Contrast-Enhanced Ultrasound and Conventional Ultrasound Characteristics of Sub-Centimeter Thyroid Nodules. Ultrasound Med Biol (2015) 41:3102-8. doi: 10.1016/j.ultrasmedbio.2015.04.026

43. Zhan J, Ding H. Application of Contrast-Enhanced Ultrasound for Evaluation of Thyroid Nodules. Ultrasonography (2018) 37:288-97. doi: 10.14366/ usg.18019

44. Chung J, Lee YJ, Choi YJ, Ha EJ, Suh CH, Choi M, et al. Clinical Applications of Doppler Ultrasonography for Thyroid Disease: Consensus Statement by the Korean Society of Thyroid Radiology. Ultrasonography (2020) 39:315-30. doi: 10.14366/usg.20072

45. Chen HY, Liu WY, Zhu H, Jiang DW, Wang DH, Chen Y, et al. Diagnostic Value of Contrast-Enhanced Ultrasound in Papillary Thyroid Microcarcinoma. Exp Ther Med (2016) 11:1555-62. doi: 10.3892/etm. 2016.3094

46. Ma JJ, Ding H, Xu BH, Xu C, Song LJ, Huang BJ, et al. Diagnostic Performances of Various Gray-Scale, Color Doppler, and ContrastEnhanced Ultrasonography Findings in Predicting Malignant Thyroid Nodules. Thyroid (2014) 24:355-63. doi: 10.1089/thy.2013.0150

47. Xu Y, Qi X, Zhao X, Ren W, Ding W. Clinical Diagnostic Value of ContrastEnhanced Ultrasound and TI-RADS Classification for Benign and Malignant Thyroid Tumors: One Comparative Cohort Study. Med (Baltimore) (2019) 98: e14051. doi: 10.1097/md.0000000000014051
48. Fang D, Ma W, Xu L, Liu Y, Ma X, Lu H. A Predictive Model to Distinguish Papillary Thyroid Carcinomas From Benign Thyroid Nodules Using Ultrasonographic Features: A Single-Center, Retrospective Analysis. Med Sci Monit (2019) 25:9409-15. doi: 10.12659/MSM.917825

49. Liu J, Zheng D, Li Q, Tang X, Luo Z, Yuan Z, et al. A Predictive Model of Thyroid Malignancy Using Clinical, Biochemical and Sonographic Parameters for Patients in a Multi-Center Setting. BMC Endocr Disord (2018) 18:17. doi: 10.1186/s12902-018-0241-7

50. Xi X, Gao L, Wu Q, Fang S, Xu J, Liu R, et al. Differentiation of Thyroid Nodules Difficult to Diagnose With Contrast-Enhanced Ultrasonography and Real-Time Elastography. Front Oncol (2020) 10:112. doi: 10.3389/ fonc. 2020.00112

Conflict of Interest: The authors declare that the research was conducted in the absence of any commercial or financial relationships that could be construed as a potential conflict of interest.

Copyright (c) 2021 Wang, Dong, Nie, Wang, Liu and Niu. This is an open-access article distributed under the terms of the Creative Commons Attribution License (CC BY). The use, distribution or reproduction in other forums is permitted, provided the original author(s) and the copyright owner(s) are credited and that the original publication in this journal is cited, in accordance with accepted academic practice. No use, distribution or reproduction is permitted which does not comply with these terms. 